\title{
Topical issue on non-isothermal transport in complex fluids
}

\author{
Published online: 2 May 2017 - (C) EDP Sciences / Società Italiana di Fisica / Springer-Verlag 2017
}

\section{Introduction}

Multicomponent fluids subjected to a temperature gradient generally undergo separation among their components, a phenomenon termed thermodiffusion in molecular fluid mixtures or thermophoresis for particles in a complex fluid, like a colloidal suspension. The physical principles determining the phenomenology of these transport processes are still not fully grasped, particularly for complex and/or multicomponent mixtures, for which even isothermal diffusion needs to be better understood. In spite of being cross transport phenomena, thermodiffusion and associated thermal forces have huge practical relevance in many fields like, among others, biophysics [1], oil industry [2], combustion and jet propulsion research [3], or even in its role related to the origin of life [4].

A continuous interaction between theory, experiments and numerical simulations is needed to further our understanding of thermodiffusion in complex systems, and the goal of the topical issue we are presenting now is to contribute to such an effort by compiling under a common umbrella a series of papers by leading scientists in the field from all over the world.

This topical issue contains several papers that were first presented at the 12th International Meeting on Thermodiffusion (IMT12) held in Madrid (Spain) on June, 2016. The continuous celebration of the IMT series of meetings, since its inception in 1994 by Prof. Jean K. Platten, has given an important boost to thermodiffusion research. A nice illustration of this fact is given by the data in fig. 1, which represents the annual distribution, according to the Web of Science ${ }^{\circledR}$ database, of scientific papers with TOPIC = THERMODIFFUSION. In the covered period 1939-2017, the total number of publications is 1495. A simple glance to the data displayed in fig. 1 shows a step-like increase in the annual number of publications coincident with the beginning of the IMT series of meetings, in 1997-2000. We wish, on behalf of the whole community, to express here our gratitude to the founders of the IMT initiative.

The last 4-6 years have shown interesting and novel tendencies in thermodiffusion research, particularly on the experimental side. We refer to the formation of relatively large research groups linked to space experiments, performed either on board the International Space Station (ISS) or in microgravity capsules launched on expendable rockets. Convection is the traditional greatest foe of accurate thermodiffusion measurements. Furthermore, complications associated to it grow larger as the number of components in the system increases, starting with ternaries. Hence, it is quite sensible to look at microgravity conditions to perform these accurate experiments, while working in space typically requires large research groups. Hence, it is not surprising at all the development in latter times of the DCMIX ${ }^{1}[5]$ and the $\mathrm{SCCO}^{2}[2]$ projects, focused on the experimental determination of thermodiffusion and/or diffusion coefficients in ternary and quaternary mixtures; as well as the forthcoming NEUF-DIX ${ }^{3}$ (now Giant Fluctuations) project [6], focused on the associated topic of non-equilibrium fluctuations induced by the Soret effect. These projects have been a driving force pushing thermodiffusion research in latter years and, on behalf of the whole community, we wish also here to thank ESA, NASA, CNSA, CSA and ROSCOSMOS for the jointly support to these initiatives.

\footnotetext{
1 Diffusion Coefficients in ternary MIXtures.

2 Soret Coefficients in Crude Oil.

3 Non-EquilibriUm Fluctuations in CompleX Systems.
} 


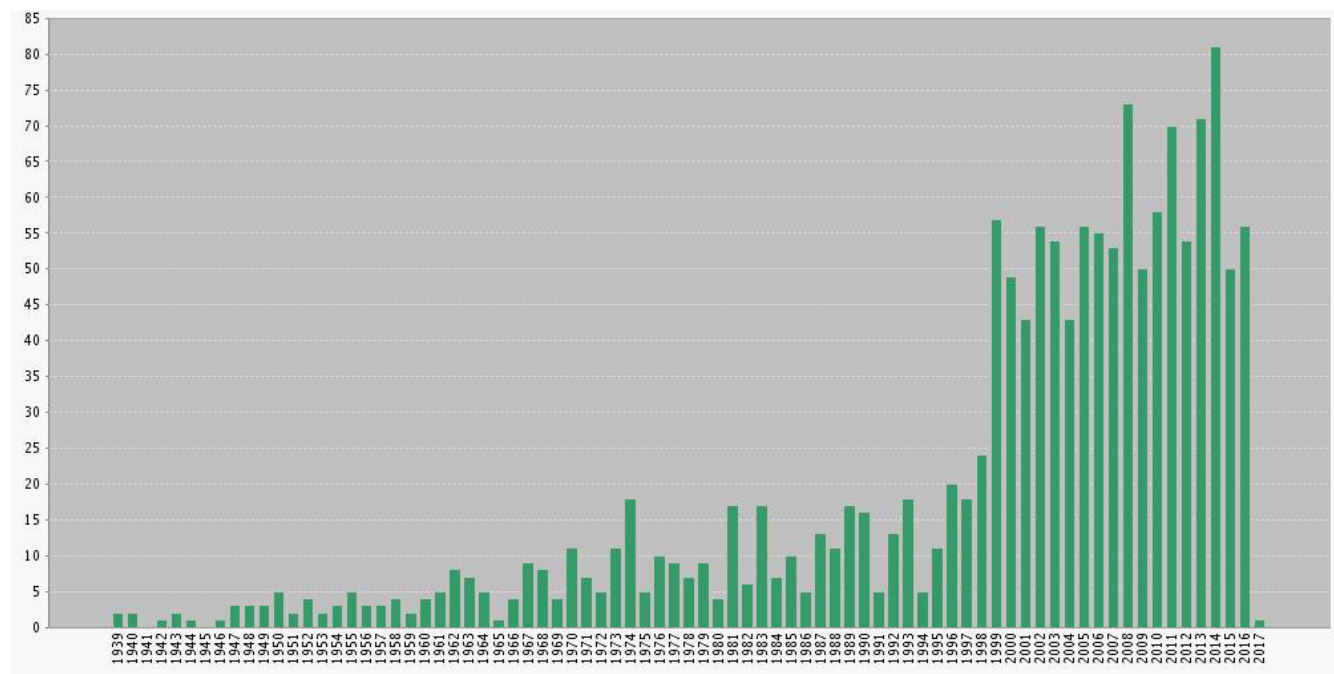

Fig. 1. Number of items published yearly with TOPIC $=$ THERMODIFFUSION according to the Web of Science ${ }^{\circledR}$ database. Since 1939 the total number of publications is 1495. Note an important enhancement around the years 1997-2000, in coincidence with the beginning of the IMT series of meetings.

\section{Topical issue}

The EPJ E topical issue Non-isothermal transport in complex fluids contains a total of twenty one scientific papers, which for ease of presentation we have here roughly divided into five groups. The various contributions are shortly presented in what follows without any specific order, simply selecting for each one the most representative group to us. Some of the papers below might be classified in several of the groups, and the current choice maybe simply reflects the personal tastes of the editors. We ask for understanding by the authors, particularly if they feel somehow misclassified.

\subsection{Diffusion and thermodiffusion in complex systems}

As expected, many papers in this topical issue deal with diffusion and thermal diffusion in various complex systems. In particular, thermal forces in polystyrene beads are investigated by Syshchyk et al. [7], while Orlishausen and Köhler [8] cover metallic particles in polymer networks and Eguchi et al. [9] oligosaccharides in polar solvents.

Also isothermal diffusion in complex systems is covered by Ancherbak et al. [10], who developed a high-pressure Taylor dispersion apparatus to experimentally measure trace diffusion in supercritical $\mathrm{CO}_{2}$. Using the same technique, but at atmospheric pressure, Rodríguez et al. [11] present trace diffusion measurements of some aminoacids in a physiological aqueous medium. Diffusion and thermal diffusion at high pressure are also investigated by Lizarraga et al. [12] who employed a different technique combining a thermogravitational column with optical shadowgraph measurements.

\subsection{Computer simulations}

Lately, computer simulations, either molecular dynamics or computational fluid mechanics (CFD), are becoming more prominent in thermodiffusion research. Indeed, during the IMT12 Conference there was a scientific session specifically devoted to molecular dynamic simulations. Such a research trend is also present in this topical issue, which includes a contribution by Hafskjold [13] who, using molecular dynamics, studies transient thermodiffusion in a binary LennardJones fluid. In addition, Mutschler et al. [14] present a CFD study of convection of a ternary mixture in a porous medium.

\subsection{Space experiments}

As already mentioned, space experiments have been playing an important role in thermodiffusion during the latest years, and it is expected that this trend will continue in the coming future. Hence Croccolo, who gave an invited plenary lecture during IMT12, publishes here in collaboration with Bataller [15] their ground results in which by reducing the thickness of a Soret cell they mimic a low-gravity environment. In addition, Baaske et al. [6] present an 
overview of the future space project NEUF-DIX, which recently entered the A/B phase after being selected by ESA to be carried out onboard the ISS in a coming round of microgravity experiments.

This topical issue also contains papers associated to the current DCMIX space experiment, thus Santos et al. [16] report diffusion coefficients measured on ground for the DCMIX-2 mixture by the Taylor dispersion method. Moreover, Bataller et al. [17] present preliminary laboratory results for one of the mixtures to be flown in the DCMIX-4 mission.

\subsection{Non-equilibrium concentration fluctuations induced by the Soret effect}

The investigation of non-equilibrium fluctuations is prominent in this topical issue, in part because of the scientific background of the editors. During IMT12 we had an invited plenary lecture by Prof. J.V. Sengers of the University of Maryland which, in this issue, appears as a Colloquium paper [18]. In addition to that, there is a contribution by Giavazzi et al. [19] who discuss the double enhancement of the concentration fluctuations in a binary liquid mixture due to the simultaneous effects of a temperature gradient and the proximity to an (equilibrium) critical point. Moreover, Martínez Pancorbo et al. [20] incorporate the effects of buoyancy in the theory of fluctuations in a ternary mixture subjected to a temperature gradient; while Oprisan et al. [21] present an experimental study of non-equilibrium fluctuations in magnetic colloidal suspensions, for which the application of an external magnetic field modifies the fluctuation intensity.

\subsection{Soret-driven convection}

Several of the papers contained in this topical issue deal with convection in binary and multicomponent systems, where the effects of thermodiffusion are quite relevant. For instance, Ryzhkov and Kozlova [22] combining theory and CFD simulations study Soret-driven separation not far from a consolute critical point of a binary mixture. Similarly, also Ouadhani et al. [23] combine theory and CFD to analyze the effects of vibration when a binary fluid is embedded in a porous medium. In addition, Lyubimova et al. [24] present a linear stability analysis, as well as non-linear amplitude equations, for a ternary mixture subjected to a temperature gradient with constant heat flux at the walls; while Smorodin et al. [25] consider convection of a colloidal suspension in a Hele-Shaw cell. Finally, we mention a second paper by Lyubimova et al. [26] where not only convection threshold, but also non-linear regimes in binary RayleighBénard convection are examined theoretically.

We want to express our thanks to all the authors and reviewers for timely submitting well-formatted contributions that simplified the editing of this topical issue of The European Physical Journal E. We are also indebted to all the journal staff for their competence and patience, with particular thanks to Solange Guéhot and to the Editors-in-Chief: Andreas Bausch and Francesco Sciortino.

\section{References}

1. C.B. Mast, D. Braun, Phys. Rev. Lett. 104, 188102 (2010).

2. G. Galliero, H. Bataller, F. Croccolo, R. Vermorel, P. Artola, B. Rousseau, V. Vesovic, M. Bou-Ali, J.M. Ortiz de Zárate, K. Zhang, F. Montel, Micrograv. Sci. Technol. 28, 79 (2016).

3. S. Palle, R.S. Miller, Combust. Flame 151, 581 (2007).

4. P. Baaske, F.M. Weinert, S. Duhr, K.H. Lemke, M.J. Russell, D. Braun, Proc. Natl. Acad. Sci. U.S.A. 104, 9346 (2007).

5. A. Mialdun, C. Minetti, Y. Gaponenko, V. Shevtsova, F. Dubois, Micrograv. Sci. Technol. 25, 83 (2013).

6. P. Baaske, H. Bataller, M. Braibanti, M. Carpineti, R. Cerbino, F. Croccolo, A. Donev, W. Köhler, J.M. Ortiz de Zárate, A. Vailati, Eur. Phys. J. E 39, 119 (2016).

7. O. Syshchyk, D. Afanasenkau, Z. Wang, H. Kriegs, J. Buitenhuis, S. Wiegand, Eur. Phys. J. E 39, 129 (2016).

8. M. Orlishausen, W. Köhler, Eur. Phys. J. E 39, 98 (2016).

9. K. Eguchi, D. Niether, S. Wiegand, R. Kita, Eur. Phys. J. E 39, 86 (2016).

10. S. Ancherbak, C. Santos, J.C. Legros, A. Mialdun, V. Shevtsova, Eur. Phys. J. E 39, 111 (2016).

11. D.M. Rodríguez, L.M.P. Verissimo, M.C.F. Barros, D.F.S.L. Rodrigues, M. Melia Rodrigo, M.A. Esteso, C.M. Romero, A.C.F. Ribeiro, Eur. Phys. J. E 40, 21 (2017).

12. I. Lizarraga, F. Croccolo, H. Bataller, M. Mounir Bou-Ali, Eur. Phys. J. E 40, 36 (2017).

13. B. Hafskjold, Eur. Phys. J. E 40, 4 (2017).

14. D. Mutschler, M.A. Larabi, A. Mojtabi, Eur. Phys. J. E 40, 49 (2017).

15. F. Croccolo, H. Bataller, Eur. Phys. J. E 39, 132 (2016).

16. C.I.A.V. Santos, V. Shevtsova, A.C.F. Ribeiro, Eur. Phys. J. E 40, 40 (2017).

17. H. Bataller, T. Triller, B. Pur, W. Köhler, J.M. Ortiz de Zárate, F. Croccolo, Eur. Phys. J. E 40, 35 (2017).

18. F. Croccolo, J.M. Ortiz de Zárate, J.V. Sengers, Eur. Phys. J. E 39, 125 (2016). 
19. F. Giavazzi, A. Fornasieri, A. Vailati, R. Cerbino, Eur. Phys. J. E 39, 103 (2016).

20. P. Martínez Pancorbo, J.M. Ortiz de Zárate, H. Bataller, F. Croccolo, Eur. Phys. J. E 40, 22 (2017).

21. A. Oprisan, A. Rice, S.A. Oprisan, C. Giraudet, F. Croccolo, Eur. Phys. J. E 40, 14 (2017).

22. I.I. Ryzhkov, S.V. Kozlova, Eur. Phys. J. E 39, 130 (2016).

23. S. Ouadhani, A. Abdennadher, A. Mojtabi, Eur. Phys. J. E 40, 38 (2017).

24. T.P. Lyubimova, E.S. Sadilov, S.A. Prokopev, Eur. Phys. J. E 40, 15 (2017).

25. B.L. Smorodin, I.N. Cherepanov, S.N. Ishutov, B.I. Myznikova, Eur. Phys. J. E 40, 18 (2017).

26. T. Lyubimova, N. Zubova, V. Shevtsova, Eur. Phys. J. E 40, 27 (2017).

Rafael Delgado-Buscalioni, Mohamed Khayet, José María Ortiz de Zárate, and Fabrizio Croccolo 